\title{
Estudo comparativo de eficácia de larvitrampas e ovitrampas para vigilância de vetores de dengue e febre amarela
}

\section{Comparative study of the efficiency of larval and ovitraps for the surveillance of dengue and yellow fever vectors}

Cristiano Correa de Azevedo Marques*, Gisela Rita de Alvarenga Monteiro Marques*, Marylene de Brito*, Luiz Gonzaga dos Santos Neto*, Vânia de Campos Ishibashi*, Francisco de Assis Gomes*

\begin{abstract}
MARQUES, C. C. de A. et al. Estudo Comparativo de eficácia de larvitrampas e ovitrampas para vigilância de vetores de dengue e febre amarela. Rev. Saúde Pública, 27: 237-41, 1993. Com a finalidade de aprimorar a vigilância entomológica dos vetores de Dengue e Febre Amarela - Aedes aegypti e Aedes albopictus - no Estado de São Paulo, Brasil, realizou-se estudo comparativo de eficácia de larvitrampas (armadilhas de larvas), e ovitrampas (armadilhas de ovos). A região estudada é infestad $\mathbf{x}$ somente pelo Aedes albopictus, espécie que conserva hábitos silvestres, mas também coloniza cu iadouros artificiais. A primeira parte do estudo foi realizada em área periurbana de Tremembé-SP, onde foram comparados três ocos de árvore, 23 ovitrampas e 5 larvitrampas. A segunda parte dos experimentos desenvolveu-se no Município de Lavrinhas-SP, no distrito de Pinheiros, onde 20 ovitrampas foram instaladas (uma por quadra) e 5 larvitrampas foram localizadas em pontos estratégicos (comércios, depósitos e postos). Os resultados obtidos mostraram que a ovitrampa, além da capacidade de positivar-se mesmo em presença de criadouros naturais, possui eficiência superior à larvitrampa. Constatou-se que para avaliação de efeitos da termonebulização as ovitrampas apresentaram uma significativa redução na média de ovos, o que não se verificou em relação ao Índice de Breteau.
\end{abstract}

Descritores: Controle de mosquitos. Ecologia de vetores. Aedes. Dengue, prevenção. Febre amarela, prevenção.

\section{Introduçáo}

O Estado de São Paulo possui atualmente 585 municípios, dos quais $74,4 \%$ estão infestados pelos vetores de Dengue e Febre Amarela (Aedes aegypti e Aedes albopictus). Atualmente a distribuição desses vetores caracterizam três áreas distintas, a saber: região oeste, onde predomina Aedes aegypti; região central, onde ocorrem ambas as espécies, e regiăo leste, onde predomina Aedes albopictus. Tal distribuição parece estar relacionada a fatores mesológicos (Buralli, Marques \& Gerola $\left.{ }^{2}, 1991\right)$.

Atualmente o Estado de São Paulo utiliza, para vigilância entomológica, basicamente dois métodos: avaliação de densidade larvária, ou Índice de

Superintendência de Controle de Endemias (SUCEN) Taubaté, SP - Brasil

Separatas/Reprints: C.C. de A. Marques - Av. Nove de Julho, 372 - 12020-200- Taubaté, SP - Brasil

Edição subvencionada pela FAPESP. Processo Medicina 93/ 0208-5
Breteau, e larvitrampas (pneus-armadilhas). Ambos os métodos são baseados na fase larvária desses culicídeos vetores, o que não necessariamente reflete a população de fêmeas potencialmente infectantes. Tal parâmetro certamente seria mais indicado pela Taxa de Picadas; que no entanto, é dificilmente exequível na rotina.

Por outro lado, podemos inferir a densidade de fêmeas pelo produto da hematofagia, ou seja, a oviposição. Vários métodos de captura de ovos têm sido testados a partir dos trabalhos de Fay \& Perryz $^{5}, 1965$ e Fay \& Eliason 6 , 1966, todos apresentando bons resultados em campo (Jakob \& Bevier9, 1969).

A região do Vale do Paraíba, onde o presente estudo foi levado a termo, situa-se em área de predominância de Aedes albopictus, espécie esta encontrada em criadouros artificiais e naturais (Gomes \& Marques ${ }^{7}, 1988$ ). Estudos posteriores realizados na região demonstraram a capacidade desse vetor em ocupar nichos vagos, colonizando criadouros naturais periurbanos, possibilitando realimentar os criadouros artificiais urbanos ou domésticos (Gomes \& col. ${ }^{8}, 1992$ ). Tais fatos motivaram 0 presente estudo, que objetiva alcançar 
formas mais eficazes de monitorar a infestação e avaliar as açôes de controle desenvolvidas.

\section{Material e Método}

A primeira parte do presente estudo desenvolveu-se em 1992, em uma chácara localizada na região periurbana do Município de Tremembe-SP. No local foram demarcados 3 ôcos de árvore, instaladas 23 ovitrampass e 5 larvitrampas. Nos ocos foram colocadas paletas de eucatex de $20 \mathrm{~cm}$ $x 05 \mathrm{~cm}$ e mantidos com água natural, as ovitrampas consistiram de vasos plásticos pretos de $15 \mathrm{~cm}$ de diâmetro por $15 \mathrm{~cm}$ de profundidade, nas quais foram instaladas as mesmas paletas utilizadas nos ocos e mantida a mesma distância entre elas e também das larvitrampas. Estas consistiram de pneus os quais eram mantidos com $1.000 \mathrm{ml}$ de água destilada.

As pesquisas foram realizadas diariamente no período da manhã, com base nas informaçð̃es do horário de oviposição de Aedes albopictus (Chadee \& Corbert ${ }^{4}, 1989$ ). Os estudos foram realizados de 7 de março a 30 de abril, período de maior atividade das espécies estudadas, segundo Gomes \& col. $^{8}, 1992$.

A segunda parte dos experimentos teve como local o Município de Lavrinhas-SP, no distrito de Pinheiros, distante $95 \mathrm{~km}$ de Taubaté, com uma população de 600 habitantes, 150 edificaçðes e 20 quadras. Nesse local foram instaladas 20 ovitrampas semelhantes ao experimento anterior, conforme as premissas de instalação de Jakob \& Bevier $^{9}$ (1969), sendo colocada uma ovitrampa por quadra.

As larvitrampas, semelhantes ao experimento de Tremembé, foram colocadas em pontos estratégicos (comércios, depósitos, postos de combustíveis...), de acordo com Jakob \& Bevier $^{9}$ (1969). Tais armadilhas foram pesquisadas semanalmente. Em concomitância foram realizados índices de densidade larvária (Índice de Breteau) (Alves \& col. ${ }^{1}, 1991$ ), nessa mesma localidade. Tais atividades foram desenvolvidas de janeiro a junho de 1992.
Acada pesquisa a paleta era identificadae acondicionada em sacos plásticos individuais e encaminhada ao laboratório para identificação e contagem dos ovos. As larvas do pneu-armadilha eram fixadas em álcool a 70\% e levadas ao laboratório para identificação e contagem. No periodo de abril e maio foram feitos 6 ciclos de termonebulização ambiental com cipermetrina à dosagem de $6 \mathrm{~g} / \mathrm{ha}$, com intervalo médio de 6 dias a cada ciclo, no distrito de Pinheiros.

Com relação à identificação dos ovos, estes eram colocados para eclodir e obter confirmação da espécie pela identificação das larvas em quarto estádio. No entanto, tal leitura das paletas não apresentou nenhuma dificuldade, sendo o índice de erro igual a zero. As identificaçőes foram feitas em microscópio esteroscópico, com aumento de 40 vezes. A única espécie de culicídeo que ocorreu concomitantemente a Aedes albopictus foi Aedes terrens, cujos ovos são facilmente diferenciados à microscopia óptica.

\section{Resultados e Discussáo}

A Tabela 1 sintetiza os resultados da primeira parte do estudo realizado no Município de Tremembé, onde se comparou dois tipos de armadilhas, além de criadouros naturais de Aedes albopictus.

Constatou-se desempenho superior da ovitrampa quando comparada ao oco de árvore e à larvitrampa, não somente quanto ao aspecto de positividade como ao número de exemplares capturados.

Outro aspecto observado diz respeito ao coeficiente de variação que, entre o criadouro natural (oco), larvitrampa e ovitrampa, esta última apresentou-se mais estável, ou seja, com menor coeficiente de variação.

Nos criadouros naturais somente foram avaliados ovos depositados nas paletas, o que não impediu que houvesse ovos depositados em outros substratos no interior do oco, porém pôde-se observar que este fato não chegou a afetar as armadilhas a ponto dessas perderem sua eficiência como instrumento de

Tabela 1. Comparação entre ovitrampas, larvitrampas e ocos para Aedes albopictus - Área experimental de Tremembé-SP, durante o período de 7 de março a 30 de abril de 1992.

\begin{tabular}{|c|c|c|c|c|c|c|c|}
\hline \multicolumn{2}{|c|}{ Criadouros } & \multicolumn{6}{|c|}{ Exames } \\
\hline \multirow[t]{2}{*}{ Tipo } & \multirow[t]{2}{*}{$N^{R}$} & \multirow[t]{2}{*}{ Realizados } & \multicolumn{2}{|c|}{ Positivos } & \multirow{2}{*}{$\begin{array}{c}\text { Média } \\
\text { exemplares } \\
\text { coletados } \\
\text { exames/dia }\end{array}$} & \multirow{2}{*}{$\begin{array}{l}\text { Desvio- } \\
\text { padrāo }\end{array}$} & \multirow{2}{*}{$\begin{array}{l}\text { Coeficiente } \\
\text { de variaçāa } \\
\text { de Pearsor }\end{array}$} \\
\hline & & & $N^{2}$ & $\%$ & & & \\
\hline $\begin{array}{l}\text { Ovitrampas } \\
\text { Larvitrampas } \\
\text { Ocos }\end{array}$ & $\begin{array}{r}23 \\
5 \\
3\end{array}$ & $\begin{array}{l}782 \\
170 \\
102\end{array}$ & $\begin{array}{r}163 \\
11 \\
12\end{array}$ & $\begin{array}{r}20,8 \\
6,5 \\
11,8\end{array}$ & $\begin{array}{r}21,9 \\
8,1 \\
2,5\end{array}$ & $\begin{array}{r}21,4 \\
21,9 \\
7,6\end{array}$ & $\begin{array}{r}97,6 \\
272,5 \\
301,7\end{array}$ \\
\hline
\end{tabular}




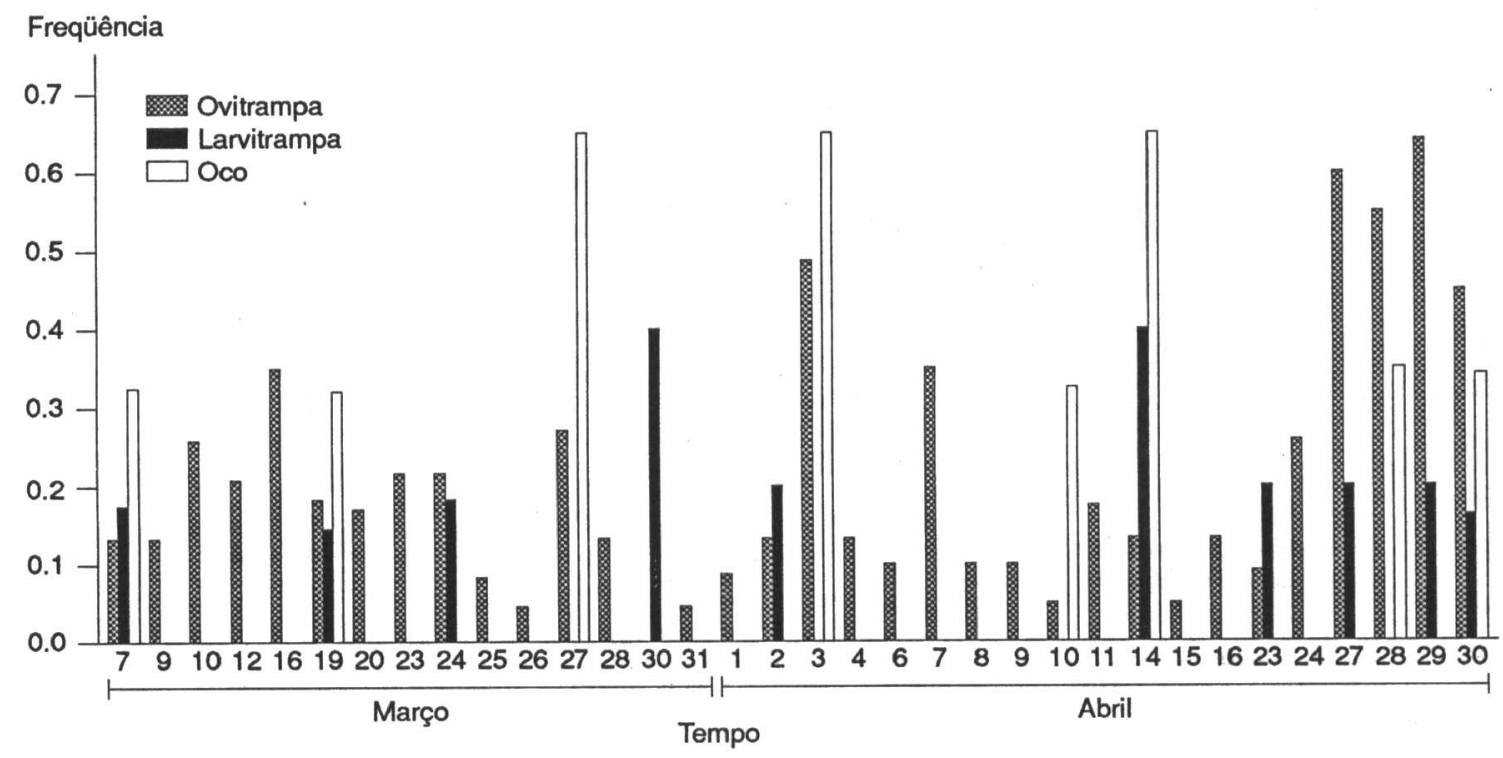

Figura 1. Freqüência de positividade de ovitrampas, larvitrampas e ocos para Aedes albopictus, área experimental de Tremembé, São Paulo, no período de 07.03.92 a 30.04.92.

Tabela 2. Comparação entre ovitrampas e larvitrampas para Aedes albopictus no distrito de Pinheiros, Município de Lavrinhas, SP, durante o período de 13 de abril a 21 de maio de 1992.

\begin{tabular}{|c|c|c|c|c|c|c|c|}
\hline \multicolumn{2}{|c|}{ Criadouros } & \multicolumn{6}{|c|}{ Exames } \\
\hline \multirow[t]{2}{*}{ Tipo } & \multirow[t]{2}{*}{$N^{2}$} & \multirow[t]{2}{*}{ Realizados } & \multicolumn{2}{|c|}{ Positivos } & \multirow{2}{*}{$\begin{array}{c}\text { Média } \\
\text { exemplares } \\
\text { coletados } \\
\text { exames/dia }\end{array}$} & \multirow{2}{*}{$\begin{array}{l}\text { Desvio- } \\
\text { padrão }\end{array}$} & \multirow{2}{*}{$\begin{array}{l}\text { Coeficiente } \\
\text { de variação } \\
\text { de Pearson }\end{array}$} \\
\hline & & & Ne & $\%$ & & & \\
\hline $\begin{array}{l}\text { Ovitrampas } \\
\text { Larvitrampas }\end{array}$ & $\begin{array}{r}19 \\
5\end{array}$ & $\begin{array}{r}114 \\
30\end{array}$ & $\begin{array}{r}104 \\
7\end{array}$ & $\begin{array}{l}91,2 \\
23,3\end{array}$ & $\begin{array}{r}41,5 \\
4,1\end{array}$ & $\begin{array}{r}23,2 \\
5,6\end{array}$ & $\begin{array}{r}55,8 \\
136,1\end{array}$ \\
\hline
\end{tabular}

$$
\%
$$

positividade

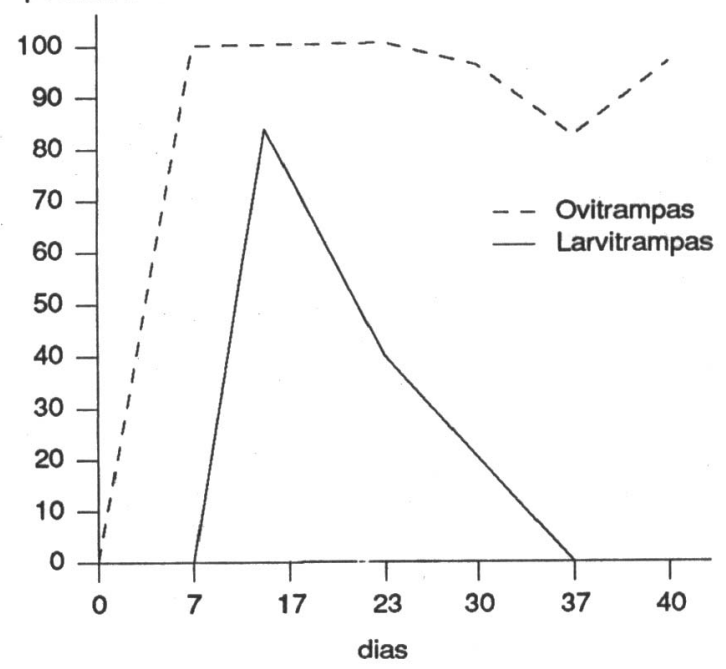

Figura 2. Positividade de ovitrampas e larvitrampas para Aedes albopictus no distrito de Pinheiros, Município de Lavrinhas, SP, no período de 13.04 .92 a 21.05 .92 . vigilância vetorial, o que para Aedes albopictus é de particular importância (Gomes e col. ${ }^{8}, 1992$ ).

Completando os resultados dos estudos realizados em Tremembé, a Figura 1 apresenta 0 desempenho diário dos três tipos de substratos oferecidos. Nele pôde-se observar a constante positividade de ovitrampa, conforme havia sido determinado pelo coeficiente de variação.

A Tabela 2 sintetiza os resultados da segunda etapa do trabalho realizado no Município de Lavrinhas, onde o mesmo padrão obtido na fase experimental, no Município de Tremembé, reproduzse, ou seja, o percentual de positividade e também o número de exemplares por armadilha foi maior nas ovitrampas.

A observação análoga para o coeficiente de variação da ovitrampa, que também foi menor que o coeficiente de variação da larvitrampa, sugere haver maior estabilidade da primeira em relação à segunda.

$\mathrm{Na}$ Figura 2, a ovitrampa se positivou mais precocemente que a larvitrampa, refletindo o bom 


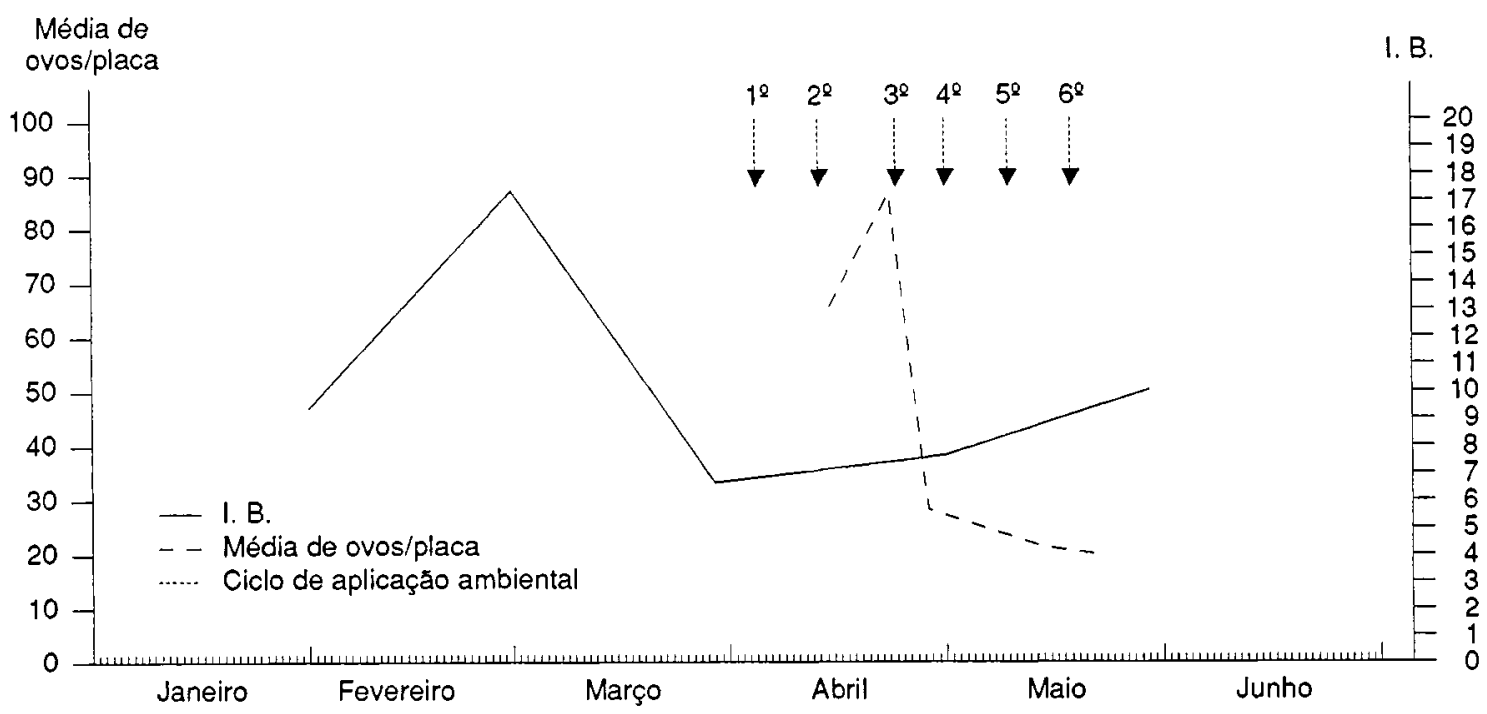

Figura 3. Densidade de ovos por ovitrampa, I. B. e ciclos de termonebulização com cipermetrina 6 g/ha em funçăo do tempo em dias.

instrumento de vigilância vetorial que poderia apresentar.

A Figura 3 apresenta os Índices de Breteau do período, os ciclos de aplicação de termonebulização com cipermetrina e a positividade de ovitrampas. Foram observados os seguintes fatos:

- A partir do segundo ciclo de termonebulização a positividade das ovitrampas reduziu-se substancialmente indicando provável redução da população de fêmeas grávidas. Inversamente, o Indice de Breteau tende a aumentar e tal resultado decorre da grande quantidade de ovos no meio ambiente, os quais não são afetados pela termonebulização.

- A ovitrampa pareceu indicar rapidamente a dinâmica da população de alados através da redução da oviposição.

- A termonebulização tem efeito temporário, caso não sejam adotadas medidas de controle ambiental, em especial à remoção de criadouros.

Tabela 3. Tabela de contingência para positividade de ovitrampas e larvitrampas - Area experimental de Tremembé - SP.

\begin{tabular}{lccc}
\hline \multirow{2}{*}{ Ovitrampas } & $(+)$ & $(-)$ & Total \\
\cline { 2 - 3 } Larvitrampas & 163 & 619 & 782 \\
\cline { 2 - 3 } Total & 11 & 159 & 170 \\
\cline { 2 - 3 }$\propto=0,05$ & 174 & 778 & $952=n$ \\
\hline
\end{tabular}

Tabela 4. Tabela de contingência para positividade de ovitrampas e larvitrampas no distrito de Pinheiros - Municipio de Lavrinhas - SP.

\begin{tabular}{lccc}
\hline \multirow{4}{*}{ Ovitrampas } & $(+)$ & $(-)$ & Total \\
\cline { 2 - 4 } Larvitrampas & 104 & 10 & 114 \\
\cline { 2 - 4 } Total & 07 & 23 & 30 \\
\cline { 2 - 4 }$\propto=0,05$ & 111 & 33 & 144 \\
\hline
\end{tabular}

Para testar a diferença entre as medidas obtidas no estudo, foi utilizado o teste de $\mathrm{X}^{2}$, onde para ambos os experimentos houve associação entre ovitrampas e maior positividade a 0,05 de significância. (Tabelas de contingência 3 e 4 ).

Conclui-se que a ovitrampa, tal como verificado por Chadee $^{3}$ (1990), apresenta-se como sendo bom instrumento de detecção precoce de vetores de Dengue e Febre Amarela, e em particular para Aedes albopictus que consegue positivar-se apesar da existência de criadouros naturais. Outra utilização importante para este tipo de armadilha está na avaliação de controle químico de alados, quando os índices de densidade larvária parecem não refletir, com rapidez e intensidade necessárias, a densidade da população vetora.

MARQUES, C.C. de A. et al. [Comparative study of the efficiency of larval and ovitraps for the surveillance of dengue and yellow fever vectors]. Rev. Saúde Pública, 
27: 237-41, 1993. A comparative study of the efficiency of ovitraps and larval-traps was undertaken with a view to improving the entomological survey of vectors of Dengue and Yellow Fever - Aedes aegypti and Aedes albopictus - in S. Paulo State, Brazil. The region studied is infested only by Aedes albopictus, a species that keeps to wild habitats but colonizes artificial breeding grounds as well. The first part of the study was located in a periurban area of Tremembé county were 3 hollon trees, 23 ovitraps and 5 larval-traps were compared. The second part of these experiments took place in Lavrinhas county (Pinheiros district), where 20 ovitraps and 5 larval-traps were tested. The results showed that the ovitrap was more efficiente than larval-traps and were positive even in the presence of natural breeding grounds. It was also observed un the evaluation of the results of "thermonebulization (fog)" that the ovitraps showed strong reduction in the average number of eggs, but this was not observed in the Breteau Index.

Keywords: Mosquito control. Ecology, vectors. Aedes. Dengue, prevention. Yellow fever, prevention.

\section{Referências Blbliográficas}

1. ALVES, M.C.G.P.; GURGEL, S.M.; ALMEIDA, M.C.R.R. Plano amostral para cálculo de densidade larvária de Aedes aegypti e Aedes albopictus no Estado de São Paulo, Brasil. Rev. Saúde Pública, 25: 251 5, 1991 .
2. BURALLI, G.; MARQUES, G.R.A.M.; GEROLA JR., O. Distribuição geográfica de Aedes aegypti e Aedes albopictus no Estado de São Paulo. Rev. Soc. Bras. Med. Trop., 24 (supl. 2): 182, 1991.

3. CHADEE, D.D. Métodos de evaluación de la población de Aedes aegypti y tratamientos con insecticidas en una población de Trinidad, Antillas. Bol. Of. Sanit. Panam., 109: 350-8, 1990.

4. CHADEE, D.D. \& CORBERT, P.S. Diel pattem of oviposition in the laboratory of the mosquito Aedes albopictus (SKUSE) (Diptera: Culicidae). Ann. Trop. Med. Parasitol.., 83: 423-9, 1989.

5. FAY, R.W. \& PERRY, A.S. Laboratory studies of ovipostional preferences of Aedes aegypti. Mosq. News, 25: 276-81, 1965.

6. FAY, R.W. \& ELIASON, D.A. A preferred oviposition site as a surveillance method for Aedes aegypti. Mosq. News, 26: 531-5, 1966 .

7. GOMES, A. de C. \& MARQUES, G.R.A.M. Encontro de criadouro natural de Aedes (Stegomyia) albopictus (SKUSE) Estado de São Paulo, Brasil. Rev. Saúde Pública, 22: 245, 1988.

8. GOMES, A.C.; FORATTINI, O.P.; KAKITANI, I.; MARQUES, G.R.A.M.; MARQUES, C.C.A.; MARUCCI, D.; BRTTO, M. Microhabitats de Aedes albopictus (SKUSE) na Região do Vale do Paraíba, Estado de São Paulo, Brasil. Rev. Saúde Pública, 26: 108-18, 1992.

9. JAkOB, W.L. \& BEVIER G.A. Application of ovitraps in the U.S. Aedes aegypti eradication program. Mosq. News, 29: 55-62, 1969.

Recebido para publicação em 15.1.1993

Reapresentado em 24.5 .1993

Aprovado para publicação em 3.6.1993 